\title{
Caracterización de los usuarios en los dispositivos de diagnóstico dual: conducta adaptativa y psicopatología
}

\section{Characterization of users in dual diagnostic devices: adaptive behaviour and psychopathology}

\begin{abstract}
Resumen
En los últimos años, fruto del trabajo de diferentes entidades para aumentar la calidad de vida de las personas con discapacidad intelectual, ha aumentado la conciencia de las administraciones acerca de las necesidades de atención de esta población en materia de salud mental. Así, desde el año 2005 han surgido centros específicos que atienden a personas con discapacidad intelectual (DI) ligera o inteligencia límite con trastornos de conducta y/o enfermedad mental asociados. Sin embargo, apenas existían datos sobre el perfil de personas atendidas, más allá de las amplias etiquetas de DI ligera/límite y alteraciones de conducta. Se realizó una recogida de datos sistemática de numerosas variables en tres centros duales (DI y graves trastornos de conducta): dos centros de día y un entorno residencial, obteniendo información de 122 personas de entre 18 y 40 años que han ocupado plaza en estos dispositivos entre 2006 y 2013. Los resultados ofrecen una visión global del perfil de usuarios ingresados, así como de las características psicopatológicas y necesidades de apoyo que presentan.
\end{abstract}

\section{Palabras clave}

Inteligencia límite, discapacidad intelectual ligera, conducta, índice problemas de conducta, psicopatología, necesidades de apoyo, diagnóstico dual.

\begin{abstract}
In the recent years, the work of different entities to increase the quality of life of people with intellectual disabilities has increased awareness of administrations about the many mental health care needs of this population. This eagerness has fostered since 2005 the establishment of specific centers that assist people with mild intellectual disability or borderline intelligence with associated behavioral and/or mental health disorders. However, there was scarce data on the profile of the assisted people, beyond the generic labels of intellectual disability/borderline intelligence and behavioral disorders. A systematic data collection was conducted, taking into account numerous variables in three dual centers (intellectual disability and severe behavioral disorders), i.e. two day-care centers and one residential center. Altogether, data have been gathered from 122 people, aged 18 to 40, who have had a place in one of these devices from 2006 to 2013. The results offer a global view of the users' profile as well as their psychopathological characteristics and need of support.
\end{abstract}

\section{Keywords}

Borderline intelligence, mild intellectual disability, behavioral alterations, behavioral problem index, psychopathology, support needs, dual diagnosis.

\section{Eduardo Guevara Cuesta <eduardo.guevara@sjd.es>}

Centro San Juan de Dios (Ciempozuelos, Madrid). España

\section{David Escudero López}

<david.escudero@funprodami.org>

Centro de día FUNPRODAMI (Madrid). España

\section{Juan Jesús Muñoz García} <juanjesus.munoz@sjd.es>

Centro San Juan de Dios (Ciempozuelos, Madrid). España

Jonás Reguilón Liébana <jonas.reguilon@alapar.org>

Fundación ALAPAR. Centro de día DIEM (Madrid). España

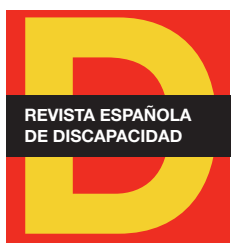

Para citar:

Guevara, E. et al. (2018):

"Caracterización de los usuarios en los dispositivos de diagnóstico dual: conducta adaptativa y psicopatología". Revista Española de Discapacidad, 6 (II): 63-80.

Doi: <https://doi.org/10.5569/23405104.06.02.04>

Fecha de recepción: 31-07-2017 Fecha de aceptación: 30-05-2018

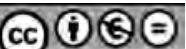




\section{Introducción}

Durante la mayor parte del siglo XX se ha producido un avance muy importante en la lucha por la igualdad de derechos de las personas, promoviendo el respeto por los derechos humanos (Asamblea General de la ONU, 1948) y fomentando la igualdad de oportunidades de colectivos especialmente desfavorecidos. En el seno de la Unión Europea se han venido desarrollando numerosas normativas dirigidas a la inclusión social de personas en riesgo de exclusión o que parten de situaciones de desventaja. Todo ello conduce a la aprobación de la Convención Internacional sobre los Derechos de las Personas con Discapacidad (Naciones Unidas, 2006), que supone un aumento importantísimo de la visibilidad de este colectivo dentro del sistema de protección de derechos humanos.

En este contexto, desde hace varias décadas existe un interés muy marcado en las instituciones europeas y nacionales por promover políticas de inclusión dirigidas hacia las personas con discapacidad para evitar la aparición de situaciones de discriminación y fomentar su participación en la sociedad como miembros de pleno derecho. Desde distintos organismos se han realizado grandes esfuerzos para que la discapacidad no sea considerada una cuestión propia del individuo sino el resultado de la interacción entre la persona y el ambiente. El cambio de paradigma que comienza a apuntarse en la Asociación Americana de Retraso Mental (AAMR, 2004) con la novena definición del concepto de retraso mental va dirigido en esa línea. Tanto la Organización Mundial de la Salud (OMS, 2001) como las revisiones de la Asociación Americana de Retraso Mental (AAMR, 2004; AAIDD, 2011), confirman esta evolución. Por otro lado, ha quedado demostrada en la literatura (Martínez-Leal et al., 2011; Salvador Carulla et al., 2007, 2009; Novell Alsina et al., 2005) la alta prevalencia de problemas de salud mental en esta población. Además, se ha descrito el llamado "efecto eclipsador", caracterizado por la tendencia a atribuir síntomas psicopatológicos a la propia discapacidad intelectual dada la mayor vulnerabilidad de esta población a sufrir problemas de salud mental (Martorell et al., 2011).

Respecto a nuestro entorno, la Unión Europea, a través de distintos programas marco, ha ido desarrollando normas que los países miembros han hecho suyas a través de la elaboración de planes específicos dirigidos a la puesta en marcha de acciones concretas en sus respectivos territorios. La Declaración de Madrid en el año 2002 con el lema "no discriminación más acción positiva es igual a inclusión social" y la proclamación del año 2003 como Año Europeo de las Personas con Discapacidad suponen una declaración de intenciones en este sentido.

En nuestro país de manera paralela se han desarrollado iniciativas tanto a nivel nacional como autonómico que han recogido esta filosofía, culminando en el año 2005 con la elaboración del $1^{\text {er }}$ Plan de Acción para Personas con Discapacidad de la Comunidad de Madrid 2005-08 (Consejería de Familia y Asuntos Sociales, Comunidad de Madrid, 2005). En este plan se recogieron las necesidades percibidas entre las personas con discapacidad de la Comunidad de Madrid y sus representantes, poniendo el foco de atención en aquellos colectivos que, por sus especiales características, contaban con unas necesidades de apoyo que, por el momento, no estaban siendo cubiertas.

El reconocimiento de las necesidades de estos grupos en algo tan básico como es su salud mental lleva a plantearse la mejora de la atención del conjunto de personas con discapacidad intelectual ligera o límite 
que, además, presentan graves trastornos de conducta. Hasta ese momento no se había planteado que estas personas precisaran unos apoyos determinados por parte de la propia administración. Por esta razón, hasta esos años, perfiles como el descrito (discapacidad intelectual ligera o inteligencia límite con graves trastornos de conducta asociados) se habían quedado fuera de la red de atención a personas con discapacidad.

Con el lanzamiento del $1^{\text {er }}$ Plan de Acción para Personas con Discapacidad de la Comunidad de Madrid (Consejería de Familia y Asuntos Sociales, Comunidad de Madrid, 2005) se abre la red de servicios sociales a este colectivo. Se comienzan a ofrecer plazas en distintos recursos tanto de atención diurna como residencial. Por su parte, la federación que aglutina a la mayor parte de entidades del sector forma un grupo de trabajo compuesto por técnicos de los propios centros que elabora una guía de evaluación e intervención de personas con discapacidad intelectual y enfermedad mental y/o problemas de conducta (Plena Inclusión Madrid, 2015).

En 2005, se abre el Centro de Día DIEM, de la Fundación ALAPAR, que ofrece plazas para personas con discapacidad intelectual ligera o inteligencia límite y trastornos de conducta o enfermedad mental asociados. El mismo año, se crean las unidades para personas con discapacidad intelectual ligera o límite y graves trastornos de conducta en los complejos hospitalarios San Juan de Dios y Benito Menni, en Ciempozuelos. En 2007 se abre el Centro de Día FUNPRODAMI, en Vallecas, ofreciendo plazas al mismo grupo de destinatarios. En 2009 se abren dos nuevas residencias (APADIS y GRUPO AMAS) que van dirigidas a la atención de personas con cualquier tipo de discapacidad intelectual con graves alteraciones de conducta y/o enfermedad mental asociada.

Por último, cabe destacar que la administración recoge como una de las acciones estratégicas del plan la colaboración entre las consejerías de Asuntos Sociales y de Sanidad. Es habitual que las personas con discapacidad intelectual sufran un efecto de "puerta giratoria", en lo que se refiere a su atención en salud mental, ya que los diferentes recursos para personas con discapacidad (gestionados desde la Consejería de Asuntos Sociales), no tienen como objetivo principal cubrir estas demandas, y desde los recursos propios de Salud Mental (Consejería de Sanidad) se les deriva a recursos de la red de atención a la discapacidad, con lo que estas necesidades quedan sin cubrir. Fruto de esta interacción nace el SESM-DI (Servicio Especializado de Salud Mental en Discapacidad Intelectual), que comienza su andadura ofreciendo sus servicios de manera ambulatoria a todos los centros que lo demanden.

Llegados a este punto, la atención a personas con discapacidad intelectual y trastornos de conducta y/o enfermedad mental asociados en la Comunidad de Madrid se puede resumir en el cuadro que aparece en la figura 1. 
Figura 1. Diagrama de la red específica de atención para personas con DI y graves trastornos de conducta en la Comunidad de Madrid.

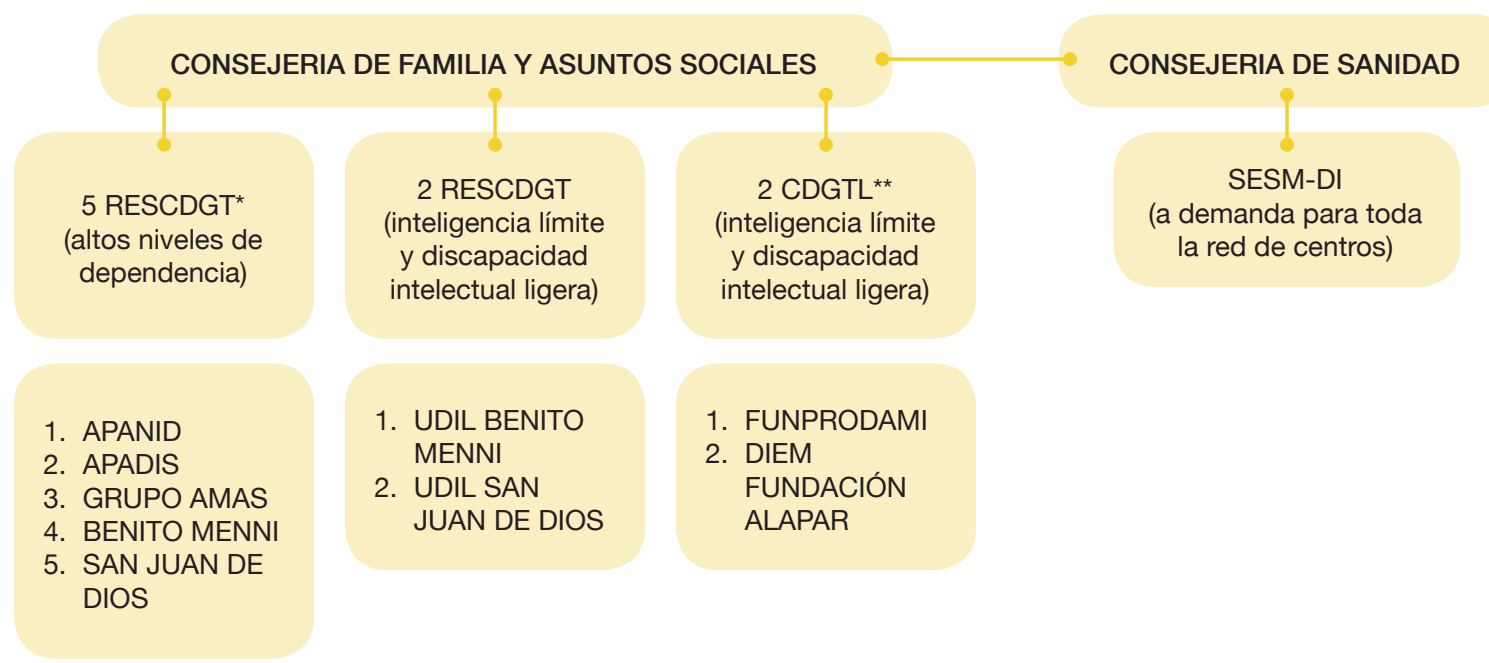

*Residencia con centro de día y atención para personas con graves trastornos de conducta.

${ }^{*}$ Centro de día con atención para personas con graves trastornos de conducta para personas con inteligencia límite.

Fuente: elaboración propia.

De las reuniones que algunos psicólogos de los recursos específicos para personas con inteligencia límite y trastornos de conducta recién creados (en adelante nos referiremos a ellos como "centros duales") celebraban para analizar las dificultades que encontraban en el quehacer cotidiano de sus propios centros, surge el Proyecto REDDUAL, cuyo trabajo exponemos en el presente artículo, centrado en usuarios de dispositivos de la Comunidad de Madrid.

No todos los centros dedicados a la atención de estas personas participan en el proyecto REDDUAL. Seguidamente se exponen aquellos recursos que sí lo hacen.

a. Centro San Juan de Dios de Ciempozuelos:

El centro San Juan de Dios está situado en la localidad de Ciempozuelos, Madrid. Cuenta con más de 120 años de experiencia. Se trata de un centro residencial, contado actualmente con 1149 plazas, de las cuales, un $42 \%$ (482) pertenecen al área de personas con discapacidad intelectual (DI). Dicha área se encuentra dividida en diferentes unidades coordinadas en una atención integral e individualizada al residente.

Dentro del proyecto REDDUAL participa la Unidad 05B, un dispositivo dirigido a personas con discapacidad intelectual ligera/inteligencia límite con graves trastornos de conducta y/o enfermedad mental asociada. Esta unidad residencial cuenta con 20 plazas para personas entre 18 y 45 años con el perfil mencionado. 
b. Centro de Día DIEM, Fundación ALAPAR (Madrid, Fuencarral-El Pardo):

La Fundación ALAPAR (antes Fundación Carmen Pardo-Valcarce) situada en el municipio madrileño de Fuencarral, lleva más de 65 años trabajando por la participación de las personas con discapacidad en la sociedad.

En el proyecto Reddual participa, el Centro de Día DIEM, que cuenta con 20 plazas concertadas con la Consejería de Asuntos Sociales. Atiende a personas de entre 18 y 40 años, con inteligencia límite o discapacidad intelectual ligera (excepcionalmente se ha atendido a algún caso con discapacidad intelectual moderada) con problemas de salud mental y alteraciones de conducta.

El centro de día DIEM ofrece un tratamiento intensivo de dos años tanto a la persona con discapacidad como a su familia, desde una perspectiva integradora.

c. El Centro de Día FUNPRODAMI (Madrid, Vallecas):

Cuenta con 20 plazas concertadas con la Consejería de Asuntos Sociales y 3 plazas privadas. Atiende a personas de entre 18 y 40 años, con el mismo perfil de personas con discapacidad que el centro anterior. La duración del tratamiento con carácter general es de dos años.

En el centro de día FUNPRODAMI se favorece la recuperación o adquisición del conjunto de habilidades y competencias personales y sociales necesarias para el funcionamiento en la comunidad.

Se constata es que no existían estudios al respecto con datos objetivos de la Comunidad de Madrid. En algunos artículos (Martínez-Leal et al., 2011; Salvador Carulla et al., 2009), guías (Martorell et al., 2011), informes (Salvador Carulla et al., 2007) y manuales (Novell Alsina et al., 2005) se muestran datos sobre la presencia de enfermedad mental y/o alteraciones conductuales en personas con discapacidad intelectual. Sin embargo, en la Comunidad de Madrid tan solo hay estimaciones al respecto (Salvador Carulla et al., 2007), situando la prevalencia de alteraciones conductuales y/o enfermedad mental en personas con discapacidad intelectual en torno al $30 \%$.

En un contexto tan amplio de trabajo se ha tratado de perfilar una serie de objetivos concretos. Estos consistieron en ofrecer datos sociodemográficos descriptivos de la población a la que atendemos en nuestros recursos. Asimismo, se pretende caracterizar a los dispositivos participantes en el estudio en función de variables como el índice de trastornos de conducta y otras vinculadas a necesidades de apoyo y psicopatología. La finalidad es ofrecer una imagen del perfil de usuarios ingresados en estos centros.

\section{Método}

\subsection{Participantes}

Los centros que participan en este estudio son los siguientes: Complejo Hospitalario San Juan de Dios, Ciempozuelos; Centro de Día DIEM, Fundación ALAPAR Madrid (Fuencarral-El Pardo); y Centro de Día FUNPRODAMI, Madrid (Vallecas). De entre ellos, la muestra participante en el presente estudio es de 122 usuarios entre 18 y 40 años (datos recogidos entre 2006 y 2013): 
- Residenciales:

- UDIL del Centro San Juan de Dios (Ciempozuelos). - 30 participantes atendidos desde 2006 hasta 2013.

- Centros de Día:

- Centro de Día DIEM de la Fundación Carmen Pardo-Valcarce (Madrid)- 64 participantes atendidos desde 2006 hasta 2013

- Centro de Día FUNPRODAMI (Madrid) - 28 participantes atendidos desde 2007 hasta 2013.

\subsection{Materiales}

La recogida de datos del presente estudio se ha llevado a cabo con entrevistas clínicas y cuestionarios administrados a usuarios y familiares por parte de los psicólogos de referencia de cada centro. Los instrumentos de medida utilizados han sido los siguientes:

- Entrevista psicosocial: elaborada para este estudio, que recoge variables sociodemográficas (edad, sexo, municipio, etc.) y relativas a la salud mental del sujeto (antecedentes familiares de enfermedad mental, diagnósticos, tratamientos recibidos, etc.). Esta entrevista se realizó tanto con el usuario/a como con su familia o cuidadores principales.

- Subescala de Problemas de Conducta del ICAP (Inventario para la Programación de Servicios y Programación Individual) (Bruininks et al., 1986; Montero Centeno, 1993). Evalúa los siguientes problemas de conducta:

- Comportamiento autolesivo o daño a sí mismo: se hace daño en su propio cuerpo. Por ej., golpeándose o dándose cabezazos.

- Heteroagresividad o daño a otros: causa dolor físico a otras personas o a animales. Por ej., golpeando, dando patadas, mordiendo.

- Destrucción de objetos: intencionalmente rompe, estropea o destruye cosas. Por ej.: golpeando, rasgando.

- Conducta disruptiva: interfiere las actividades de otros. Por ej., abrazándose en exceso a otros, acosándoles o importunándoles.

- Hábitos atípicos y repetitivos (estereotipias): son conductas poco usuales, extrañas, que se repiten una y otra vez. Por ej. ir y venir por la habitación, balancearse.

- Conducta social ofensiva: son conductas que ofenden a otros. Por ej.: hablar en voz muy alta y/o blasfemar.

- Retraimiento o falta de atención: son problemas de falta de relación con otros o de no prestar atención. Por ej.: mantenerse alejado de otras personas, expresar temores poco corrientes, mostrarse muy inactivo y demostrar muy poca concentración.

- Conductas no colaboradoras: son conductas en las que la persona no colabora. Por ej.: negarse a obedecer, no hacer sus tareas o no respetar las reglas.

Consta en total de 8 ítems, uno por cada problema de conducta y dos escalas de respuesta para cada una: frecuencia (escala de respuesta de 0 =Nunca a $5=$ Una o más veces en una hora) y gravedad (escala de respuesta de $0=$ No es grave a $4=$ Extremadamente grave). 
Tras su puntuación y corrección ofrece cuatro índices de problemas de conducta: Índice Interno, Índice Asocial, Índice Externo e Índice General. Estos índices se puntúan de +10 (Normal) a -41 o menos (Muy Grave).

Este cuestionario se aplicó en todos los centros que han participado en el estudio. Fue respondido por los profesionales que atienden a los usuarios.

- Mini PAS-ADD. Escala de Evaluación para la Detección de los Problemas de Salud Mental de las Personas Adultas con Retraso Mental (García, 2009). Proporciona información sobre síntomas psiquiátricos a profesionales (trabajadores sociales, psicólogos, personal de enfermería, etc.) con el fin de facilitar la toma de decisiones acerca de posibles derivaciones a otros servicios u otros tipos de intervención especializada. Forma parte de la batería PAS-ADD.

Consta de 86 ítems y su escala de respuesta presenta cuatro opciones (No ha sucedido durante las últimas cuatro semanas, Leve, Moderado o Grave).

Las puntuaciones finales se dividen en 6 subescalas: depresión, ansiedad, hipomanía, trastorno obsesivo-compulsivo, psicosis, y trastorno no especificado.

El Mini Pas-Add se aplicó en todos los centros que han participado en el estudio y fue respondido por los familiares y/o profesionales de la persona con DI.

- SIS Escala de Intensidad de Apoyos (Verdugo et al., 2007). Este instrumento evalúa las necesidades de apoyo de personas con discapacidad intelectual. Analiza 49 tipos de actividades de la vida diaria agrupadas en seis escalas: Vida en el hogar (8 ítems), Vida en la comunidad (8 ítems), Aprendizaje a lo largo de la vida (9 ítems), Empleo (8 ítems), Salud y seguridad (8 ítems) y Actividades sociales (8 ítems). Además, presenta una Escala Suplementaria de Actividades de Protección y Defensa ( 8 ítems) y otra de Necesidades Excepcionales de Apoyo Médico (16 ítems) y de Apoyo Conductual (13 ítems). Evalúa la frecuencia, el tiempo y el tipo de apoyo con escalas de respuesta tipo Likert de 1 a 4.

\subsection{Diseño}

Se realizó un estudio descriptivo transversal con los datos disponibles procedentes del protocolo comentado. Las variables del análisis descriptivo se presentan en la tabla 1. 


\begin{tabular}{l}
\hline Tabla 1. Variables de la investigación \\
\begin{tabular}{|l|l|}
\hline Variables del estudio & Usuario adoptado \\
\hline Tipo de centro & Discapacidad intelectual \\
Fecha de ingreso & Porcentaje de discapacidad \\
Fecha de nacimiento (año) & Situación legal \\
Edad de ingreso & Diagnóstico psiquiátrico de Centro Base -servicios \\
Sexo & especializados de atención básica a personas con discapacidad \\
Lugar de residencia & intelectual- \\
Código postal & Diagnóstico psiquiátrico del propio centro de diagnóstico dual \\
Municipio & Patología orgánica (relacionada con la DI: problemas \\
Distrito & neurológicos, epilepsia, síndromes genéticos...) \\
Procedencia & Causa de la DI \\
Atención previa & Presencia de TGD \\
Atención previa diurna social & Tratamiento farmacológico inicial \\
Atención previa diurna sanitaria & Tratamiento farmacológico actual \\
Seguimiento psiquiátrico & Urgencias psiquiátricas en el año previo al ingreso en nuestro \\
Seguimiento psicológico & centro \\
Antecedentes psiquiátricos familiares & Ingresos psiquiátricos en el año previo al ingreso en nuestro \\
Antecedentes DI en la familia & centro \\
Antecedentes TGD en la familia & \\
Antecedentes de problemas neurológicos en la familia & \\
\hline
\end{tabular}
\end{tabular}

Fuente: elaboración propia.

\subsection{Procedimiento}

Establecemos a continuación los siguientes periodos de desarrollo del estudio:

- Fase 1: Reuniones trimestrales de los profesionales de los centros.

- Definición y puesta en común de variables a incluir en el estudio.

- Creación de una base de datos común en SPSS 23.0.

- Establecimiento de objetivos.

- Fase 2: Administración de entrevistas y cuestionarios.

- Parte del proceso habitual de evaluación de los centros (a excepción de los centros residenciales).

- En torno a los 3 primeros meses de estancia del usuario en el centro.

- Fase 3: Introducción de datos en la base de datos.

- Fase 4: Análisis de datos: realizándose dos tipos de análisis tras la descripción inicial de la muestra.

- Se realizó un análisis descriptivo de las diferencias existentes entre los dispositivos de estudio (de carácter residencial frente a centros de día) en los diagnósticos establecidos por diferentes entidades de la red pública.

- Se realizaron diferentes ANOVAs multifactoriales partiendo, como factores, del tipo de dispositivo de ingreso y la presencia o ausencia de problemas de conducta y siendo las variables medidas 
diferentes dimensiones de la conducta adaptativa y aspectos de gravedad psicopatológica. En esta parte de la investigación, uno de los dos centros de día de la muestra no aporta datos de las dimensiones de conducta adaptativa.

- Fase 5: Conclusiones.

\section{Resultados}

\subsection{Descriptivos muestrales}

En relación a usuarios atendidos dentro de centros de diagnóstico dual, se encontró que un 17,20\% de los usuarios accede a nuestro centro de manera directa, sin proceder de ningún otro recurso de la red de atención a personas con discapacidad y no habiendo recibido atención en ningún momento de su vida por recursos especializados en discapacidad intelectual pertenecientes a la Consejería de Políticas Sociales y Familia. Asimismo, se observó que un elevado porcentaje de usuarios era procedente de centros educativos $(29,5 \%)$ y que un $12,3 \%$ de los usuarios no ha sido atendido por ningún recurso previo, incluyendo a los servicios de sanidad o sociales (figura 3).

\section{Figura 3. Procedencia previa al ingreso}

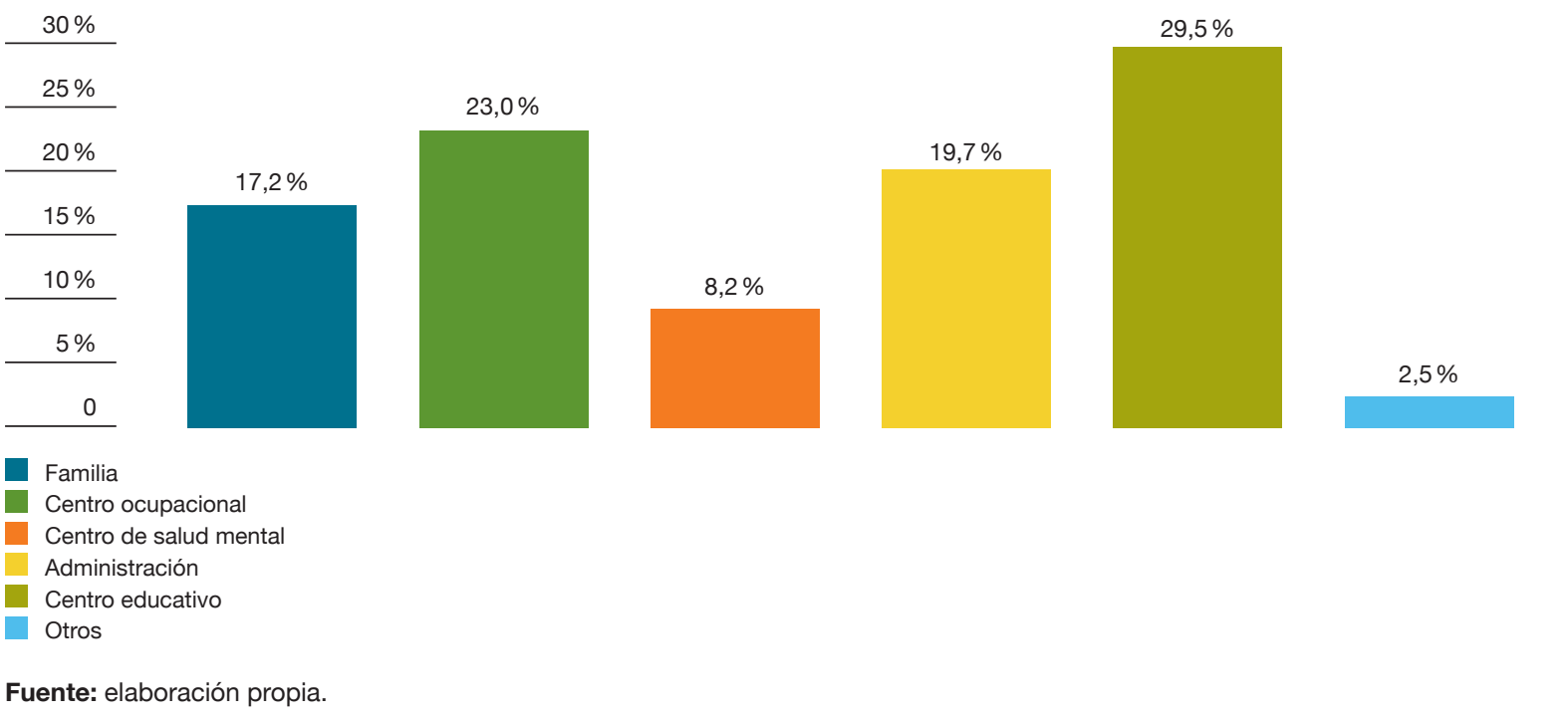

En relación a la atención previa diurna social recibida en el último año, un 21,3\% de los usuarios que cursan plaza en el centro dual no ha asistido a ningún recurso de la red de servicios sociales en el último año (figura 4). Además, existe un alto porcentaje de usuarios procedente de centros de la red de discapacidad intelectual (68,7\%). En cuanto a atención previa diurna sanitaria recibida en el último año, un 45,9\% de los usuarios que cursan plaza en el recurso dual ha pasado por una unidad hospitalaria de salud mental en el último año (figura 5). 
Figura 4. Atención previa diurna social
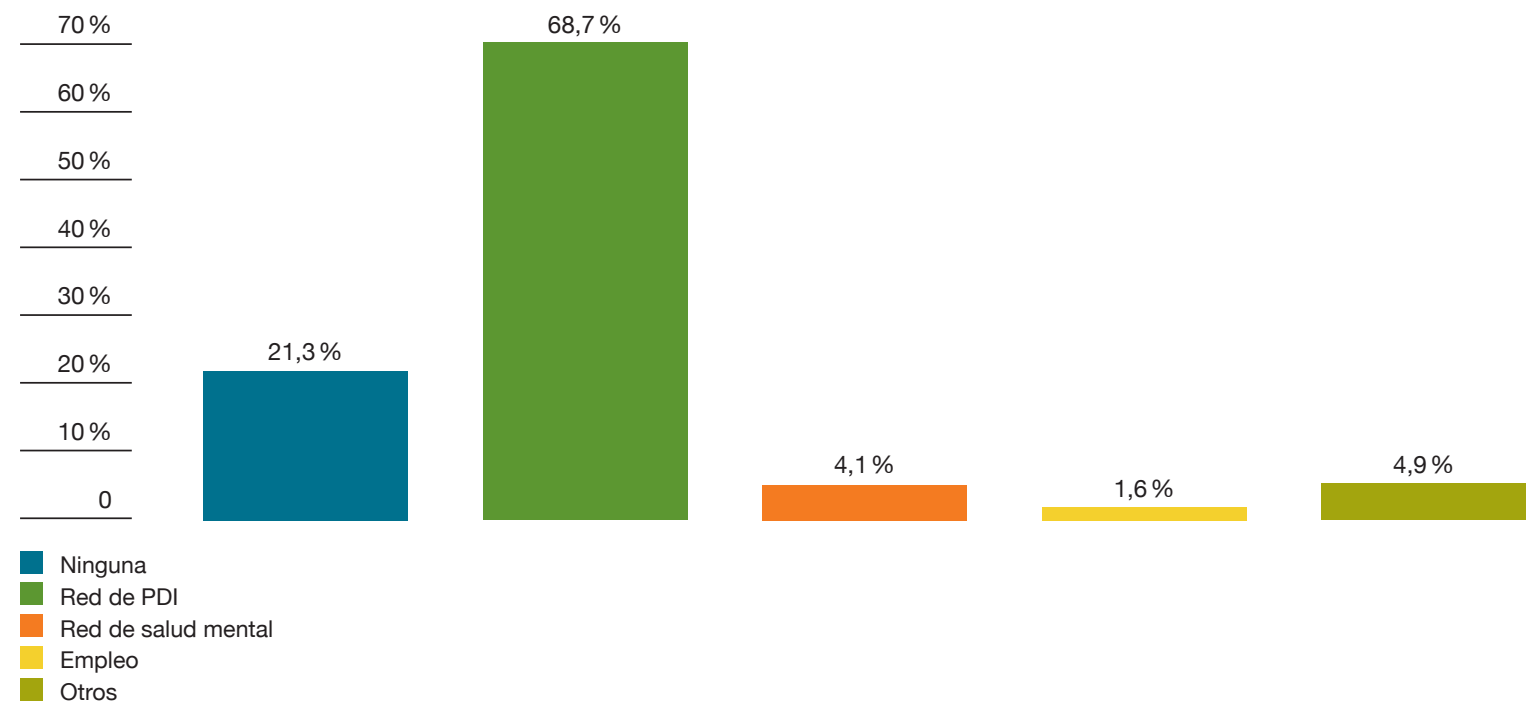

Fuente: elaboración propia.

Figura 5. Atención previa diurna sanitaria

\begin{tabular}{c}
$50 \%$ \\
\hline $40 \%$ \\
\hline $30 \%$ \\
\hline $20 \%$ \\
\hline $10 \%$ \\
\hline 0 \\
\hline Ninguna \\
Hospital de día \\
Centro de día \\
Otros
\end{tabular}

Fuente: elaboración propia.

Respecto al seguimiento psiquiátrico/psicológico previo al recurso dual, un $78,7 \%$ recibe o ha recibido tratamiento por parte del Centro de Salud Mental, mientras que un 36,1\% de los usuarios no ha recibido atención/tratamiento psicológico. Cabe destacar la elevada aparición de antecedentes de trastornos psiquiátricos en la familia (figura 6). 
Figura 6. Porcentaje de antecedentes familiares psiquiátricos y de discapacidad intelectual respectivamente

$\begin{array}{r}90 \% \\ \hline 80 \% \\ \hline 70 \% \\ \hline 60 \% \\ \hline 50 \% \\ \hline 40 \% \\ \hline 30 \% \\ \hline 20 \% \\ \hline 10 \% \\ \hline 0\end{array}$

- Sí

Desconocido

Fuente: elaboración propia.

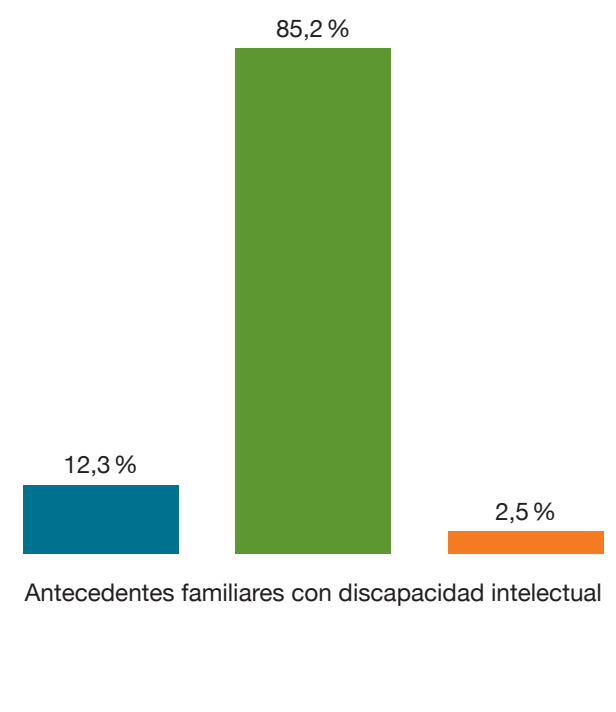

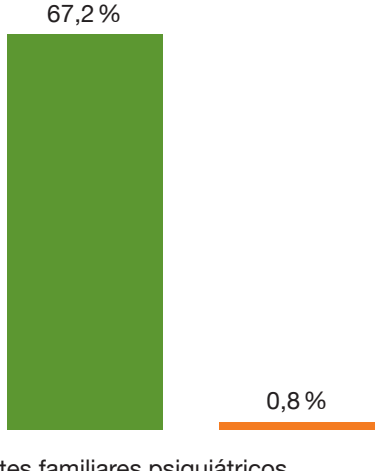

Antecedentes familiares psiquiátricos tual

\subsection{Comparativa inter-dispositivos en variables diagnósticas}

Se han realizado análisis preliminares para valorar la influencia de diferentes variables sociodemográficas en la posible existencia de diferencias significativas entre los dispositivos analizados. En este sentido, no hay diferencias significativas en la edad al ingreso de los usuarios entre los centros de día y el entorno residencial $\left(28,33\right.$ vs. 27,$\left.42 ; t_{120}=0,71 ; p \leq 0,481\right)$ pero sí en la existencia de antecedentes psiquiátricos familiares (25,3\% vs. 53,3\%; $\left.x^{2}{ }_{1}=8,13 ; p \leq 0,01\right)$, de discapacidad intelectual $\left(46,7 \%\right.$ vs. $\left.27,6 \% ; x^{2}=7,81 ; p \leq 0,01\right)$ y de trastorno neurológico $\left(6,6 \%\right.$ vs. $\left.20 \% ; x^{2}=4,54 ; p \leq 0,05\right)$.

Tomando en consideración el diagnóstico psiquiátrico establecido en el centro de referencia (se ha procedido a la agrupación de los centros de día en una única categoría frente al entorno residencial), ya sea entorno residencial o centros de día, se han encontrado diferencias significativas entre algunos de los diagnósticos establecidos y no así en la proporción global de diagnósticos. Respecto al diagnóstico de trastornos de ansiedad, el entorno residencial registra una mayor proporción de casos frente a los centros de día (58,1\% vs. $\left.6,6 \% ; x_{1}=33,52 ; p \leq 0,001\right)$, algo extensible a los trastornos del estado de ánimo $\left(26,7 \%\right.$ vs. $4,4 \% ; x^{2}=$ $16,48 ; p \leq 0,001)$ y los trastornos de conducta $\left(93,5 \%\right.$ vs. $\left.50,5 \% ; x^{2}=25,1 ; p \leq 0,001\right)$. No ocurre así en el caso de los trastornos del espectro psicótico, trastornos de la personalidad, trastornos generalizados del desarrollo y otros trastornos (categoría miscelánea).

Cuando el diagnóstico es establecido por el centro base también han aparecido diferencias significativas entre algunos de los realizados mientras que no se dan en la proporción global de diagnósticos. Atendiendo a los trastornos de ansiedad, el entorno residencial registra una mayor proporción de casos frente a los centros de día $\left(33,3 \%\right.$ vs. 8,8\%; $\left.\chi^{2}=10,17 ; p \leq 0,001\right)$, algo extensible a la categoría de otros trastornos 
$\left(29,6 \%\right.$ vs. $\left.7,7 \% ; x^{2}=9,03 ; p \leq 0,01\right)$ y la de trastornos de conducta $\left(88,9 \%\right.$ vs. $\left.34,1 \% ; x^{2}{ }_{1}=25,15 ; p \leq 0,001\right)$. No existen diferencias significativas en el caso de los trastornos del espectro psicótico, trastornos de la personalidad, trastornos del estado de ánimo y trastornos generalizados del desarrollo.

Asimismo, el diagnóstico realizado por el centro de salud mental a los participantes de los dispositivos analizados también arroja resultados con diferencias significativas en cuanto a la proporción de personas diagnosticadas frente a las que no reciben ningún diagnóstico, siendo mayor la tendencia en aquellos que están en un entorno residencial frente al centro de día (100\% vs. $\left.73,6 \% ; x_{1}{ }_{1}=10,17 ; p \leq 0,001\right)$. Ciñéndonos a las categorías específicas hay mayor proporción de diagnósticos de trastornos de ansiedad (48,4\% vs. 4,4\%; $\left.\mathrm{X}^{2}{ }_{1}=34,03 ; p \leq 0,001\right)$, de conducta $\left(80,6 \%\right.$ vs. $\left.22 \% ; x^{2}=25,15 ; p \leq 0,001\right)$ y de trastornos de la personalidad $\left(38,7 \%\right.$ vs. $\left.15,14 \% ; x^{2}{ }_{1}=7,5 ; p \leq 0,01\right)$.

La representación gráfica de los porcentajes de usuarios que reciben diagnóstico en los dispositivos del estudio se presenta en la figura 7.

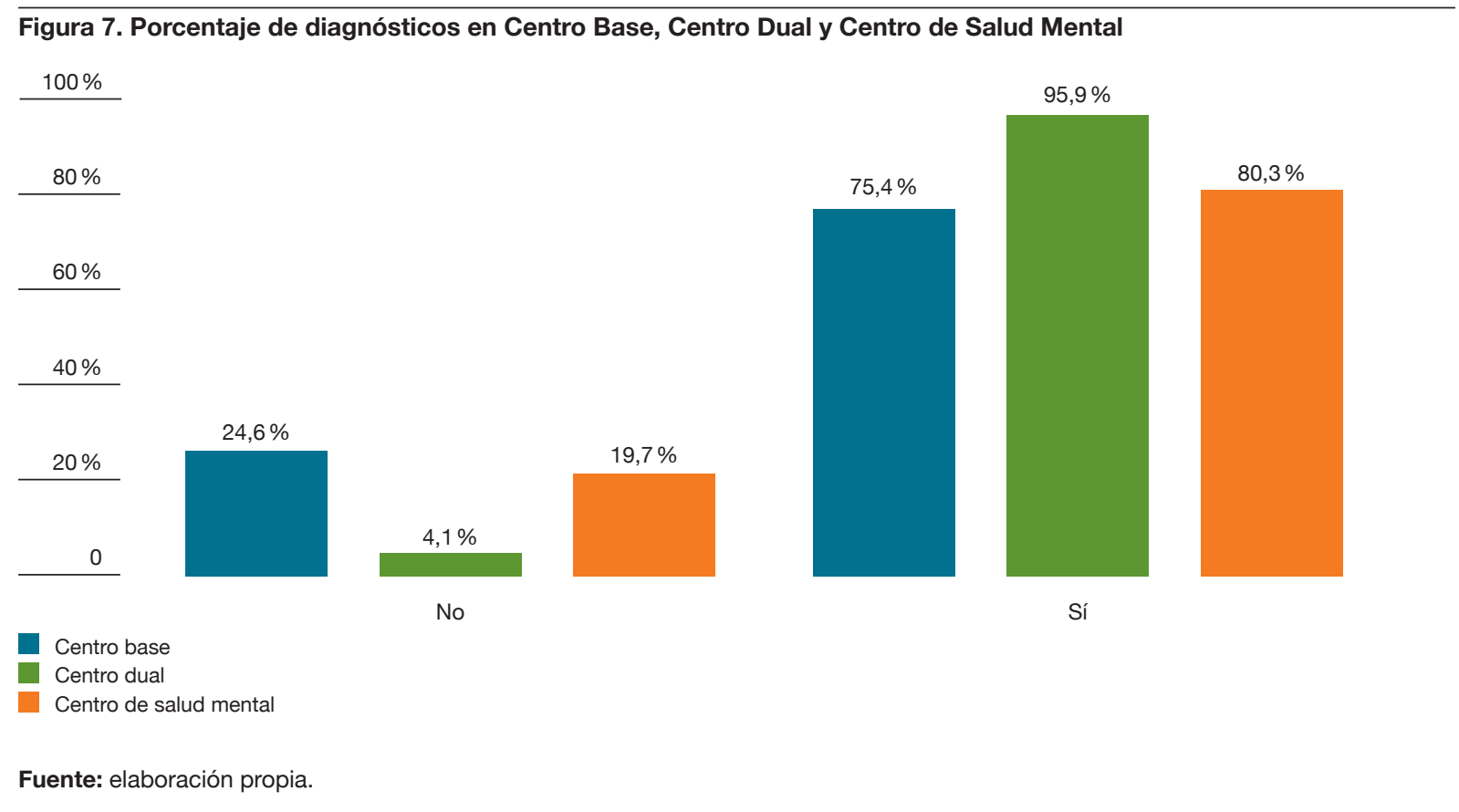

Por otro lado, se han encontrado diferencias significativas entre los dispositivos analizados en relación al número de urgencias e ingresos psiquiátricos en el año previo al ingreso, siendo mayor las proporciones en entorno residencial frente a centros de día tanto en urgencias ( $63,5 \%$ vs. $\left.33 \% ; \chi^{2}{ }_{1}=12,49 ; p \leq 0,001\right)$ como en ingresos (50\% vs. $\left.28,6 \% ; \chi^{2}{ }_{1}=6,57 ; p \leq 0,01\right)$.

Respecto al tratamiento farmacológico que reciben los participantes, en la actualidad se han encontrado diferencias significativas entre los dispositivos analizados en la proporción de pauta de benzodiacepinas $\left(87,1 \%\right.$ vs. $\left.38,5 \% ; X^{2}=21,88 ; p \leq 0,001\right)$, estabilizadores del ánimo $\left(83,9 \%\right.$ vs. $\left.59,3 \% ; x^{2}=6,16 ; p \leq 0,05\right)$ y 
otros fármacos -alta heterogeneidad en esta indicación- $\left(22,6 \%\right.$ vs. 6,6\%; $\left.\chi_{1}^{2}=6,21 ; p \leq 0,05\right)$. Estas diferencias siempre serían a favor del entorno residencial. No han aparecido diferencias significativas en antipsicóticos y antidepresivos.

Por último, tomando en consideración la gravedad de los problemas de conducta al ingreso en relación con el tipo de centro se encontró, según el ICAP (problemas de conducta), una tendencia -no significativa- $\left(x^{2} 4=\right.$ $8,62 ; p=0,07)$ a una mayor gravedad. Esta tendencia se observa en una mayor presencia de casos de problemas de conducta normales $(41,9 \%$ vs. $26,7 \%)$ o levemente graves $(45,2 \%$ vs. $33,3 \%)$ en los centros de día, mientras que, sobre todo, los casos moderadamente graves $(26,7 \%$ vs. $3,2 \%)$ son más habituales en el entorno residencial.

\subsection{Comparativa inter-dispositivos en variables relacionadas con necesidades de apoyo y gravedad psicopatológica}

De cara a la caracterización de los usuarios ingresados en un entorno residencial frente a un centro de día, se ha considerado valorar la influencia de la interacción de esta variable con la ausencia o presencia de problemas de conducta en el ICAP sobre las diferentes dimensiones de la escala de intensidad de apoyos (SIS). Se han encontrado algunas diferencias significativas que se detallan a continuación, pero, la mayoría de las mismas, proceden del perfil de usuario ingresado en cada dispositivo específico.

En este sentido, los usuarios ingresados en entorno residencial que tienen problemas de conducta tendrían menos necesidades de apoyo que los que también presentan estas dificultades en centro de día en las dimensiones de la SIS vinculadas a aprendizaje $\left(7,55\right.$ vs. 36,50; $\left.F_{1}=190,83 ; p \leq 0,05\right)$, empleo $\left(8,25\right.$ vs. $39 ; F_{1}=$ 208,68; $p \leq 0,05)$ y el área social $\left(8,60\right.$ vs. 23,$\left.17 ; F_{1}=98,68 ; p \leq 0,05\right)$. Por añadidura, la influencia aislada de la variable tipo de centro deriva en diferencias significativas en todas las dimensiones de la escala SIS y siempre con la interpretación de mayores necesidades de apoyo en los centros de día: vida en el hogar (8,41 vs. 28,13; $\left.F_{1}=1683,79 ; p \leq 0,05\right)$, vida en la comunidad $\left(8,22\right.$ vs. 21,$\left.63 ; F_{1}=626,21 ; p \leq 0,001\right)$, aprendizaje $(7,11$ vs. 32,88; $\left.F_{1}=2365,69 ; p \leq 0,001\right)$, empleo (7,96 vs. 35,38; $\left.F_{1}=2692,53 ; p \leq 0,001\right)$, salud y seguridad $(8,07$ vs. 39; $\left.F_{1}=23,88 ; p \leq 0,001\right)$, social $\left(8,22\right.$ vs. $\left.20,50 F_{1}=461,84 ; p \leq 0,001\right)$ y necesidades de apoyo -puntuación global- $\left(89,89\right.$ vs. 93,$\left.25 ; F_{1}=32,98 ; p \leq 0,001\right)$. Por último, relativo a la influencia por separado de la variable ausencia o presencia de trastornos de conducta se han encontrado diferencias significativas, siendo superiores las necesidades de apoyo en aquellos que tienen problemas de conducta -obviamente- en las dimensiones aprendizaje $\left(14,23\right.$ vs. 9,44; $\left.F_{1}=190,83 ; p \leq 0,05\right)$, empleo $\left(15,35\right.$ vs. $\left.11 ; F_{1}=208,68 ; p \leq 0,05\right) y$ social $\left(11,96\right.$ vs. 8,$\left.33 ; F_{1}=98,68 ; p \leq 0,05\right)$ de la SIS.

Finalmente, se ha realizado el mismo análisis, pero esta vez considerando como variable dependiente el Min-Pas Add y, más concretamente, las dimensiones psicopatológicas. En la interacción entre la variable tipo de centro e ICAP (presencia vs. ausencia problemas de conducta) no han aparecido diferencias significativas, algo extensible a la variable relacionada con el ICAP cuando se valora en exclusividad y, prácticamente, a la variable centro, si bien ahí han aparecido dos diferencias significativas en lo relacionado con la gravedad de la hipomanía $\left(5,70\right.$ vs. 2,$\left.12 ; F_{1}=74,69 ; p \leq 0,05\right)$ y de las psicosis $\left(0,60\right.$ vs. 21,$63 ; F_{1}=0,08$; $\mathrm{p} \leq 0,05)$. Estas diferencias sitúan una mayor gravedad de estos problemas en los usuarios ingresados en centro de día frente a los que están en un entorno residencial. 


\section{Discusión y conclusiones}

Con los resultados obtenidos en este estudio se evidencia un mejor encuadre diagnóstico en los centros duales, lo que permite una intervención más específica y ajustada, economizando recursos de la sociedad. Cabe señalar que el estudio tiene diferentes limitaciones y, entre ellas, está la de la atribución de los resultados hallados a la influencia de las variables tratadas y no otras. En este sentido, la unidad residencial sólo tiene perfiles masculinos y la existencia de perfiles femeninos en los centros de día puede determinar diferencias. Lo mismo ocurriría con el hallazgo de mayor proporción de usuarios con familiares afectados por antecedentes psiquiátricos y trastornos neurológicos en el entorno residencial frente a los centros de día que, sin embargo, tendrían mayor proporción de familiares con antecedentes de afectación por discapacidad intelectual. Estos datos podrían condicionar las diferencias halladas y/o, en su caso, ser determinantes para las mismas. Otras dificultades y limitaciones del estudio tienen que ver con el diagnóstico asignado a los participantes al estar basado en criterios clínicos de diferentes evaluadores. Por añadidura a esta variabilidad, el tamaño muestral es limitado para la generalización de los resultados.

Centrándonos en las diferencias entre los dispositivos del estudio, se observa que el entorno residencial tiene mayor proporción de trastornos de ansiedad, del estado de ánimo y de conducta, mientras que en el entorno de atención diurna se concentran más casos de psicosis e hipomanía.

También se observa un mayor número de ingresos psiquiátricos o visitas a urgencias de esta índole en el año previo al ingreso en entorno residencial, así como una mayor pauta de benzodiacepinas, estabilizadores del ánimo u otros fármacos en estos recursos.

Por otro lado, se ha encontrado una presencia de mayores necesidades de apoyo en áreas como aprendizaje, empleo o la social en personas vinculadas a centro de día frente al entorno residencial.

No encontramos diferencias significativas entre los dispositivos analizados en relación a los niveles de gravedad de los problemas de conducta, atendiéndose desde los distintos dispositivos a personas con graves trastornos de conducta y necesidades de apoyo, avalando la idea de que estos dispositivos, tanto de atención diurna como residenciales, funcionan como recursos específicos para usuarios con intensas necesidades de apoyo. En la tabla 2 se presenta un resumen de los resultados obtenidos al comparar los dos tipos de dispositivos de este estudio. 


\begin{tabular}{|c|c|c|c|c|c|}
\hline & $\begin{array}{l}\text { Diagnósticos (centros } \\
\text { duales, centro de } \\
\text { salud mental y centro } \\
\text { base) }\end{array}$ & $\begin{array}{l}\text { Gravedad } \\
\text { psicopatológica }\end{array}$ & $\begin{array}{l}\text { Ingresos } \\
\text { psiquiátricos y } \\
\text { urgencias }\end{array}$ & $\begin{array}{l}\text { Pauta } \\
\text { farmacológica }\end{array}$ & $\begin{array}{l}\text { Necesidades } \\
\text { de apoyo en } \\
\text { usuarios con } \\
\text { problemas de } \\
\text { conducta }\end{array}$ \\
\hline $\begin{array}{l}\text { Centros } \\
\text { de día }\end{array}$ & & $\begin{array}{l}\text { >Gravedad de } \\
\text { hipomanía y psicosis }\end{array}$ & & & $\begin{array}{l}\text { > Necesidades } \\
\text { de apoyo en } \\
\text { aprendizaje, } \\
\text { empleo y área } \\
\text { social }\end{array}$ \\
\hline $\begin{array}{l}\text { Unidad } \\
\text { residencial }\end{array}$ & $\begin{array}{l}\text { > Trastornos de } \\
\text { ansiedad, trastornos } \\
\text { del estado de } \\
\text { ánimo, trastornos } \\
\text { de la personalidad } \\
\text { y trastornos de } \\
\text { conducta }\end{array}$ & & $\begin{array}{l}\text { > Número de } \\
\text { urgencias } \\
\text { e ingresos } \\
\text { psiquiátricos en } \\
\text { el año previo al } \\
\text { ingreso }\end{array}$ & $\begin{array}{l}\text { >Pauta de } \\
\text { benzodiacepinas, } \\
\text { estabilizadores } \\
\text { del ánimo y otros } \\
\text { fármacos }\end{array}$ & \\
\hline
\end{tabular}

Fuente: elaboración propia.

Por otra parte, hemos obtenido resultados que, en principio, podrían parecer contradictorios con lo esperable al analizar las necesidades de apoyo en los usuarios que presentaban problemas de conducta ingresados en dos de los dispositivos del estudio (residencial vs. centro de día). Así, la presencia de mayores necesidades de apoyo en áreas como aprendizaje, empleo o la social en personas vinculadas a centro de día supone un dato sobre el que se pueden hacer diferentes interpretaciones. Inicialmente, se puede plantear una obvia limitación del estudio y que son las diferentes inter-jueces en la administración de las pruebas. Otro planteamiento podría ser que haya un sesgo optimista y/o pesimista en los mismos, de tal forma que en el entorno residencial se valora desde una perspectiva que ya no piensa en las mismas necesidades de cara a vivir en el medio natural; frente al centro de día, que puede orientar sus evaluaciones bajo el prisma de una excesiva escasez de habilidades en los usuarios para precisamente estar adaptados al medio. No podemos olvidar que quizá se pueda dar a la inversa y el entorno residencial aporte una estructura y soportes idóneos para los usuarios lo que minimiza o atenúa las necesidades de apoyo frente al centro de día donde, independientemente de la calidad de las intervenciones, puede haber un entorno menos estructurado en el que las necesidades de apoyo se hagan más patentes.

En cuanto al diagnóstico, los centros base se muestran como el dispositivo que menos diagnósticos establece a la población de estudio frente a los centros duales y los centros de salud mental. Esto se puede explicar desde el mayor seguimiento que desde los centros duales se tiene de estas personas, así como de una atención más especializada centrada en sus necesidades de salud mental, mientras que el contacto desde los centros base y los centros de salud mental, es más esporádico.

Con todo ello, los resultados de nuestro estudio nos muestran que desde estos recursos se atiende a unos usuarios que llegan a la red de diagnóstico dual con unas grandes dificultades de adaptación, que reciben una atención especializada que permite visibilizar estas dificultades.

Por otro lado, otra de las conclusiones básicas a las que alude el estudio es que estos usuarios hacen uso con amplios periodos de ingreso de plazas de Unidades de Hospitalización Breve de los hospitales de la Red 
Pública de Salud Mental. Por dicho motivo, y evidenciada la efectividad de los recursos duales para la atención de esta población, afirmamos que los centros de atención a personas con discapacidad intelectual y graves trastornos de conducta/enfermedad mental son necesarios para atender las necesidades actuales de la sociedad. La demanda es notoria y actualmente existe una amplia lista de espera en el acceso a dichas plazas.

Actualmente, la demanda de la sociedad está por encima de los recursos duales existentes. Sugerimos la ampliación de esta red para resolver la crítica situación de este colectivo y sus familiares. Pero el trabajo de los gestores no solo debe ir dirigido a abordar el momento actual, sino que también sería necesaria la creación de servicios de prevención especializados en trastornos de conducta/enfermedad mental en centros ocupacionales y centros de día, incluso implicando a la Consejería de Educación para atender lo más inmediatamente posible la aparición de las alteraciones de conducta en los centros de educación especial. Conviene señalar que la existencia de los centros de educación especial y su progresivo abandono, en detrimento de programas de inclusión educativa, abre el debate sobre la idoneidad de unos dispositivos frente a otros, si bien este debate no es objeto de este estudio.

En la misma línea, buscando una prevención y abordaje temprano en situaciones agudas, deberíamos dotar a nuestra comunidad de unidades especializadas de atención a adolescentes con discapacidad intelectual/ inteligencia límite y graves trastornos de conducta/problemas de salud mental en la sanidad pública. Y en lo referido a usuarios adultos de este perfil, dada la amplia lista de espera actual en los recursos residenciales, disponer de recursos de hospitalización breve específicos para abordar situaciones de urgencia probablemente mejoraría la calidad y efectividad de la intervención y economizaría gastos públicos en la Consejería de Sanidad a medio y largo plazo.

En cuanto a nuevas líneas de investigación, son muchos los datos que todavía se desconocen en la Comunidad de Madrid. Sería deseable saber cuál es la situación de partida en la que se encuentra la región. Es decir, conocer datos reales de prevalencia de alteraciones de conducta y/o enfermedad mental en personas con discapacidad intelectual. Con esta información y una planificación adecuada se podrían asignar recursos que hiciesen frente a las necesidades no cubiertas y que están suponiendo unos costes muy elevados a la administración regional a nivel educativo, sanitario y social.

Otra línea de investigación iría encaminada a conocer el impacto de las intervenciones sobre la calidad de vida de las personas con alteraciones de conducta/enfermedad mental y discapacidad intelectual asociadas. También sería deseable conocer qué tipo de alteraciones conductuales y/o enfermedad mental tienen un pronóstico más difícil y cuáles son los factores que están interviniendo en ello, poniendo especial énfasis en los aspectos biológicos (por ejemplo, presencia de síndromes concretos, epilepsia, trastornos cognitivos...), pero sin olvidar otros aspectos médicos, psiquiátricos, psicológicos, sociales, físicos, etc.

En definitiva, pese a las limitaciones de este estudio, que se ha centrado en ofrecer un perfil descriptivo de los usuarios ingresados en dos tipos de recursos, conviene seguir profundizando en este campo. Son muchas las líneas de investigación que se abren de cara al futuro. Si cada parte de este gran puzle que configura la atención a personas con discapacidad intelectual y alteraciones de conducta y/o enfermedad mental asume su cuota de responsabilidad en este asunto, todos (personas con discapacidad, familiares, profesionales, entidades, Administración...) nos beneficiaremos de los resultados y podremos realizar intervenciones mejores que redundarán positivamente en la calidad de vida de las personas con las que trabajamos. 
AAIDD (2011): Discapacidad intelectual: definición, clasificación y sistemas de apoyo. Madrid: Alianza Ensayo.

AAMR (2004): Retraso mental: definición, clasificación y sistemas de apoyo (traducción de Verdugo, M. A. y Jenaro, C.), Madrid: Alianza Editorial.

Asamblea General de la ONU (1948). Declaración Universal de los Derechos Humanos. Resolución 217 A (III), París, Francia.

Bruininks, R. et al. (1986): ICAP. Inventory for client and agency planning. Examiner's Manual. Allen: DLM Teaching Resources.

Consejería de Familia y Asuntos Sociales, Comunidad de Madrid (2005): Plan de acción para personas con discapacidad 2005-08. Madrid: Comunidad de Madrid.

García, R. (2009): Adaptación española del PAS-ADD 10 (Psychiatric Assessment Schedule for Adults with Developmental Disability) [Tesis doctoral no publicada]. Cádiz: Universidad de Cádiz.

Luckasson, R. et al. (1997): Retraso Mental. Definición, clasificación y sistema de apoyo. Madrid: Alianza.

Martínez-Leal, R. et al. (2011): "La salud en personas con discapacidad intelectual en España: Estudio europeo POMONA-II". Revista de Neurología, 53: 406-414.

Martorell, A. et al. (2011): Discapacidad Intelectual y salud mental. Guía práctica. $2^{\mathrm{a}}$ ed. Madrid: Consejería de Asuntos Sociales, Comunidad de Madrid.

Montero Centeno, D. (1993): Evaluación de la conducta adaptativa en personas con discapacidades. Adaptación y validación del ICAP. Bilbao: Mensajero.

Moss S, G. D. et al. (1995): The Psychiatric Assessment Schedule for Adults with a Developmentl Disability (PAS$A D D)$. Manchester: Hester Adrian Research Centre-Institute of Psychiatry.

Naciones Unidas (2006): Convención Internacional sobre los Derechos de las Personas con Discapacidad. Nueva York: ONU.

Novell Alsina, R. et al. (2005): Salud mental y alteraciones de la conducta en las personas con discapacidad intelectual. Guía práctica para técnicos y cuidadores. $3^{\mathrm{a}}$ ed. Madrid: FEAPS.

OMS (2001): Clasificación Internacional del Funcionamiento de la Discapacidad y de la Salud: CIF. Madrid: Ministerio de Trabajo y Asuntos Sociales, Instituto de Migraciones y Servicios Sociales.

Plena Inclusión Madrid (2015): Discapacidad intelectual y salud mental. Evaluación e intervención psicológica. Análisis de casos (en línea). <https://plenainclusionmadrid.org/wp-content/uploads/2017/12/DiscapacidadIntelectual-y-Salud-Mental-Evaluacion-e-intervencion-psicologica-An-lisis-de-casos.pdf>, acceso 5 de julio de 2018.

Salvador Carulla, L. et al. (2009): “Trastornos psiquiátricos en retraso mental: evaluación y diagnóstico". Psiquiatria.com, 3 (4).

Salvador Carulla, L. et al. (2007): Trastornos de la salud mental en personas con discapacidad intelectual: declaración FEAPS e informe técnico. Madrid: FEAPS. 
Verdugo, M. A. (2009): Escala Integral. Evaluación objetiva y subjetiva de la calidad de vida de personas con discapacidad intelectual. Madrid: CEPE.

Verdugo, M. A. et al. (2007): Escala de Intensidad de Apoyos SIS. Manual. Adaptación española. Madrid: TEA. 